\title{
Prevalence of Transmitted HIV-1 Drug Resistance Remains Low in Guangxi, China, Eight Years after Scale-Up of Highly-Active Antiretroviral Therapy
}

\author{
Guojian Li $^{\mathrm{a}}$ Shujia Liang ${ }^{\mathrm{b}}$ Tim J. Harrison $^{c}$ Zhenzhu Tang ${ }^{\mathrm{b}}$ Zhiyong Shen ${ }^{\mathrm{b}}$ \\ Xueyan Wang ${ }^{b}$ Xinghua Wu ${ }^{b}$ Wei Liu $^{b}$ Fuxiong Liang ${ }^{b}$ Liushuai Feng $^{b}$ \\ Jinye Yang ${ }^{b}$ Zhongliao Fang ${ }^{b}$ \\ ${ }^{a}$ Department of Public Health of Guangxi Zhuang Autonomous Region, and ${ }^{b}$ Guangxi Zhuang Autonomous Region \\ Center for Disease Prevention and Control, Nanning, China; ' ${ }^{\mathrm{D}}$ ivision of Medicine, UCL Medical School, London, UK
}

\section{Key Words}

Human immunodeficiency virus type 1 . Transmitted drug resistance $\cdot$ Highly-active antiretroviral therapy . Epidemiology · Guangxi

\begin{abstract}
Objectives: Highly-active antiretroviral therapy (HAART) was scaled up in Guangxi, China in 2005. The number of individuals receiving free HAART increased dramatically from June 2010 under the Guangxi Government's anti-HIV programme. We aimed to determine the prevalence of HIV-transmitted drug resistance (TDR) of Guangxi. Methods: HIV-positive, antiretroviral-naive individuals were recruited from the east (Hezhou), south (Qinzhou), west (Baise), north (Guilin) and centre (Laibin) of Guangxi. The pol gene of the virus from the individuals was analysed. Results: The overall prevalence of HIV TDR was 3.2\% $(7 / 216,95 \% \mathrm{Cl} 0.9-5.5)$. The prevalence rates in Baise, Guilin, Hezhou, Qinzhou and Laibin are 4.9\% (2/41, $95 \% \mathrm{Cl}-1.7$ to 11.5$), 2.3 \%(1 / 44,95 \% \mathrm{Cl}-2.1$ to 5.7$)$, $4.7 \%(2 / 43,95 \% \mathrm{Cl}-1.6$ to 11.0$), 2.6 \%(1 / 38,95 \% \mathrm{Cl}-2.5$ to $7.7)$ and $2.0 \%(1 / 50,95 \% \mathrm{Cl}-1.9$ to 5.9$)$, respectively. No significant difference in the prevalence was found among them. No factors were found to be associated with TDR, including CD4 cell counts, viral loads and genotypes. The subtypes CRF01_AE, CRF07_BC, CRF08_BC and B were found. Subtype
\end{abstract}

CRF08_BC is the predominant subtype in Baise while CRF01_ $A E$ is the predominant subtype elsewhere in Guangxi. Conclusions: The prevalence of TDR in antiretroviral-naive patients in Guangxi remains low 8 years after scale-up of HAART.

(c) 2014 S. Karger AG, Basel

Human immunodeficiency virus type 1 (HIV-1) infection remains a major global health problem. Worldwide, 34.0 (31.4-35.9) million people were living with $\mathrm{HIV}$ at the end of 2011. An estimated $0.8 \%$ of adults aged 15-49 years worldwide are living with HIV [1]. HIV originated from multiple zoonotic transmissions of simian immunodeficiency virus (SIV) from non-human primates to humans in West and Central Africa around 100 years ago. It is characterized by its enormous genetic variability, which results from the high mutation and recombination rates of the reverse transcriptase enzyme that lacks a proofreading mechanism, together with high rates of virus replication [2] and this leads to the accumulation of mutations that confer resistance to antiretroviral (ARV) drugs, during suboptimal ARV therapy (ART) [3].

The health and quality of life of patients infected with HIV/AIDS have dramatically improved since the intro-

\section{KARGER}

E-Mail karger@karger.com

www.karger.com/int (c) 2014 S. Karger AG, Basel

0300-5526/14/0575-0270\$39.50/0
Zhongliao Fang

The Guangxi Zhuang Autonomous Region Center for

Disease Prevention and Control

18 Jin Zhou Road, Nanning 530028 (China)

E-Mail zhongliaofang@ hotmail.com 
duction of highly-active antiretroviral therapy (HAART) in 1996 [4]. At the end of 2011, more than 8 million people were receiving ART in low- and middle-income countries, which was 26 times higher than the number in December 2003 [5]. However, one of the major drawbacks of ART is that it has resulted in transmitted drug resistance (TDR) [4]. For individuals, emergence of TDR can restrict subsequent ARV treatment choices and can exhaust therapeutic options, resulting in HIV-1 disease progression and death [6]. TDR is also a public health concern because it has the potential to compromise ART at the population level [7].

Guangxi Zhuang Autonomous Region, a Chinese province with a population of 51 million, is located in the southern China on the border with Vietnam. It is also a neighbour of Yunnan province, where the total number of accumulated HIV/AIDS cases is highest among the various provinces in China [8]. Guangxi started to monitor HIV/AIDS in 1986. Up to 1992, only 4 HIV cases were found by testing sera from 108,659 individuals. All of these cases were individuals from outside China. Early in 1993, 1 individual in Guangxi was found to be positive for HIV but he was not a resident of Guangxi, although he was Chinese [9]. Guangxi residents were first found to be HIV-positive in 1996. The prevalence is $20.3 \%$ among injecting drug users in Pingxiang City, on the border with Vietnam [10]. Since that time, increasing numbers of HIV/AIDS cases have been identified throughout Guangxi. By the end of 2009, Guangxi was ranked second among provinces in China in terms of the total number of accumulated HIV/AIDS cases [8]. In order to control the rapid spread of HIV, the Guangxi Government started a 5 -year period anti-HIV programme in June 2010 and this seeks to ensure best efforts to identify $\mathrm{HIV}$-infected individuals and provide free ART to all who are eligible.

Regular, population-based assessments of transmission of drug-resistant strains is important to assure continued efficacy of the limited ARV regimens in use in resource-limited counties because this can generate important information to guide first-line ART selection, support education and prevention programmes, and promote the rational use of ARV drugs by clinicians and policymakers $[11,12]$. China's National Free Antiretroviral Treatment Programme (NFATP) was launched in 2002 and scaled up in Guangxi at the beginning of 2005. Under the programme, all patients met the treatment criterion of CD4 cell count $<200 / \mathrm{mm}^{3}$ or World Health Organization (WHO) stage III or IV diseases are eligible for free six ARV drugs including azidothymidine (AZT), didanosine (DDI), stavudine (D4T), lamivudine (3TC), nevirapine (NVP) and indinavir (IDV). The number of individuals infected with HIV and receiving ART dramatically increased from June 2010 under the local government's 5-year anti-HIV programme. However, the prevalence of transmitted HIVDR had not been ascertained. Now, we have carried out a cross-sectional study to determine the prevalence of HIVDR and associated factors among ARV-naive patients throughout Guangxi.

\section{Materials and Methods}

Patient Population and Sample Design

Individuals who were confirmed as HIV antibody-positive but had never been exposed to ARVs were recruited between December 2012 and January 2013 from the HIV Voluntary Testing and Counseling Centre at the Center for Diseases Prevention and Control (CDC) of the east, south, west, north, and centre of Guangxi province. Demographic data were collected through direct application of a questionnaire before sample donation. A 9-ml venous blood was drawn from each individual. Informed consent in writing was obtained from each individual. Ethics committee approval for this study was obtained from the Guangxi Institutional Review Board. The study was conducted according to the principles of the Declaration of Helsinki.

\section{CD4 Estimation and Viral Load Testing}

CD4 counts were measured in whole fresh blood within $24 \mathrm{~h}$ by the BD FACSCount ${ }^{\mathrm{TM}}$ System (Becton Dickinson, Franklin Lakes, N.J., USA). Plasma HIV-1 RNA copy numbers were determined on thawed plasma with real-time Nucleic Acid Sequence Based Amplification (NASBA) (NucliSense Easy Q; bioMérieux, Marcy l'Etoile, France) or COBAS (Roche Applied Science, Penzberg, Germany). All measurements were performed in the local CDCs according to the manufacturers' protocols.

Extraction, PCR Amplification and Sequencing of HIV-1 RNA and DNA

Viral RNA was extracted from $140 \mu \mathrm{l}$ of HIV-1-positive plasma samples using QIAamp Viral RNA mini kits (Qiagen, Hilden, Germany) according to the manufacturer's protocol. The pol gene (protease, PR, and reverse transcriptase, RT) was amplified by RTPCR using nested PCR. The protocol is $50^{\circ}$ for $30 \mathrm{~min}, 94^{\circ}$ for $2 \mathrm{~min} ; 94^{\circ}$ for $30 \mathrm{~s}, 55^{\circ}$ for $50 \mathrm{~s}, 72^{\circ}$ for $90 \mathrm{~s}, 30$ cycles. The first round primers are:

DR2-1-CNBC: TGGAAATGTGGAAAAGAAGGAC, HXB2 2029-2050.

DR2-2-CNBC: CTGTATTTCAGCTATCAAGTCTTTTGAT GGG, HXB2 3539-3509.

The second round PCR was carried out on $5 \mu \mathrm{l}$ of the first round products in a $50-\mu \mathrm{l}$ reaction. The protocol is $94^{\circ}$ for $30 \mathrm{~s}, 55^{\circ}$ for $50 \mathrm{~s}, 72^{\circ}$ for $90 \mathrm{~s}, 30$ cycles. The primers are DR2-3-CNBC: CAGAGCCAACAGCCCCACCA, HXB2 2147-2166. DR24-CNBC: CTGCCAATTCTAATTCTGCTTC, HXB2 3462-3441. PCR Taq were products of TaKaRa Biotechnology Co. Ltd (Dalian, China). PCR products from the second round were confirmed by 
Table 1. Baseline clinical and biological characteristic of the study subjects

\begin{tabular}{|c|c|c|c|}
\hline Variables & Total $(n=216)$ & Male $(\mathrm{n}=151)$ & Female $(n=65)$ \\
\hline \multicolumn{4}{|l|}{ Age, years } \\
\hline Mean \pm SD & $44.4 \pm 15.1$ & $45.6 \pm 15.2$ & $41.6 \pm 14.5$ \\
\hline Median (range) & $42(14-82)$ & $44(14-82)$ & $39(18-71)$ \\
\hline \multicolumn{4}{|l|}{ CD4 T-cell count, cells/ $\mu \mathrm{l}$} \\
\hline Mean \pm SD & $272.1 \pm 212.0$ & $250.5 \pm 208.4$ & $381.0 \pm 221.3$ \\
\hline Median (range) & $247.5(0-980)$ & $229(0-980)$ & $297(0-896)$ \\
\hline \multicolumn{4}{|l|}{ Viral loads, RNA copies/ml } \\
\hline Mean \pm SD & $135,819.4 \pm 278,303.4$ & $134,561.4 \pm 230,125.9$ & $139,051.4 \pm 356,922.9$ \\
\hline Median (range) & $44,000(50-2,300,000)$ & $45,305(50-1,800,000)$ & $29,000(130-2,300,000)$ \\
\hline \multicolumn{4}{|l|}{ Employment, \% (n) } \\
\hline Employed & $16.7(36)$ & $15.9(24)$ & $18.5(12)$ \\
\hline Farmer & $53.7(116)$ & $53.6(81)$ & $53.9(35)$ \\
\hline Unemployed/unknown & $29.6(64)$ & $30.5(46)$ & $27.7(18)$ \\
\hline \multicolumn{4}{|l|}{ Marital status, \% (n) } \\
\hline Married & $63.4(137)$ & $62.3(94)$ & $66.2(43)$ \\
\hline Single/divorced/widowed & $33.3(72)$ & $33.8(51)$ & $32.3(21)$ \\
\hline Unknown & $3.2(7)$ & $4.0(6)$ & $1.5(1)$ \\
\hline \multicolumn{4}{|l|}{ HIV transmission route, $\%(\mathrm{n})$} \\
\hline Heterosexual & $90.3(195)$ & $87.4(132)$ & $96.9(63)$ \\
\hline MSM & $1.4(3)$ & $2.0(3)$ & 0 \\
\hline Drug injection & $5.6(12)$ & $7.3(11)$ & $1.5(1)$ \\
\hline Other/unknown & $2.8(6)$ & $3.3(5)$ & $1.5(1)$ \\
\hline \multicolumn{4}{|l|}{ Education, \% (n) } \\
\hline Primary school or less & $44.4(96)$ & $43.7(66)$ & $46.2(30)$ \\
\hline Junior high school or more & $55.6(120)$ & $56.3(85)$ & $53.8(35)$ \\
\hline \multicolumn{4}{|l|}{ Infection stage, \% (n) } \\
\hline HIV & $56.5(122)$ & $50.3(76)$ & $70.8(46)$ \\
\hline AIDS & $43.5(94)$ & $49.7(75)$ & $29.2(19)$ \\
\hline
\end{tabular}

agarose gel electrophoresis and the positive amplicons were sent to Beijing Biomed Co. Ltd (Beijing, China) for sequencing using a BigDye Terminator V3.1 Cycle Sequencing kit (Applied Biosystems, Foster City, Calif., USA).

\section{Drug Resistance Analysis}

The sequences obtained above were submitted to the Stanford HIV drug resistance database. Drug resistance mutations (DRMs) profile and ARV susceptibility were inferred using the WHO 2009 list of mutations for surveillance of TDR as implemented in the Calibrated Population Resistance tool (v5.0 beta) [13] (http://hivdb. stanford.edu).

\section{Genotyping Methods}

Genotype was determined by the NCBI viral genotyping tool (http://hiv-web.lanl.gov/content/index) using the sequences obtained above.

\section{Statistical Analysis}

Baseline clinical and biological characteristics of the study subjects were summarized as frequency (\%) for the categorical variables, and mean \pm SD or median (range) for quantitative variables. The $95 \%$ confidence interval (CI) for the prevalence of TDR was estimated. Statistical comparisons of the prevalence of TDR be- tween men and women and across the different age groups and different areas were performed using $\chi^{2}$ or Fisher's exact tests. All $p$ values were two-tailed and $p<0.05$ was considered to be significant. Logistic regression analysis was carried out to determine associations between possible risk factors and TDR. Statistical analysis was carried out using EPI Info version 6.1 and SPSS version 16.0 software.

\section{Results}

\section{Baseline Characteristics}

A total of 254 patients from five sampling sites were recruited into the study. 216 of them were positive for HIV RNA, including 151 male and 65 female study subjects (table 1). The mean ages of the male and female study subjects was 45.6 (95\% CI 30.4-60.8) and 41.6 (95\% CI 27.1-56.1) years, respectively. The median ages of the males and females were 44 (14-82) and 39 (18-71) years, respectively. $33.3 \%$ of the study subjects were single and $63.4 \%$ were married. $90.3 \%$ of them gave a history of het- 
Fig. 1. Prevalence of transmitted HIV drug resistance in various cities in Guangxi.

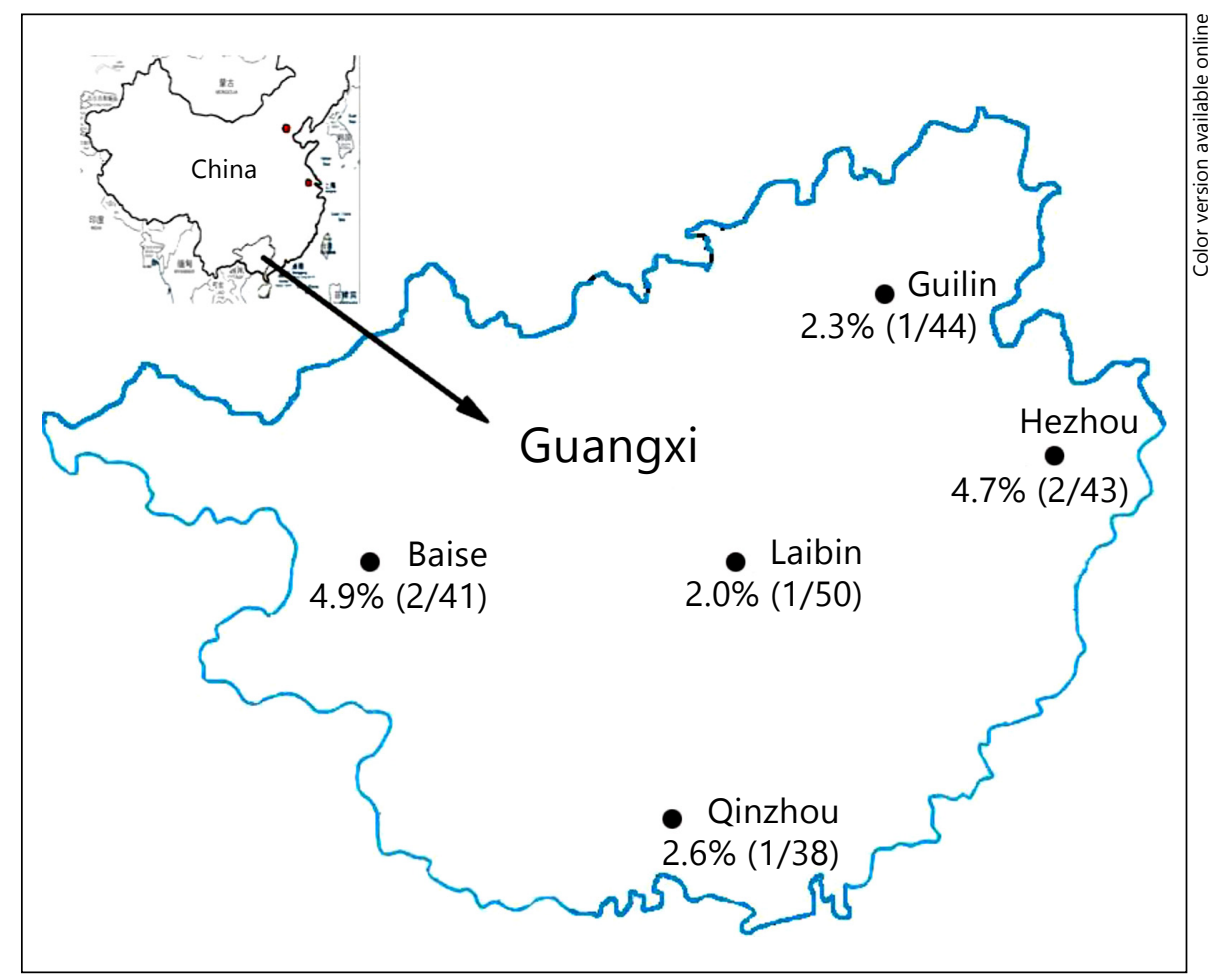

erosexual exposure, $1.4 \%$ were men who had sex with men, 5.6\% had a history of drug injection and the rest did not reveal their HIV exposure history.

At enrolment, $56.5 \%$ of them were HIV-infected while the remainder (43.5\%) were diagnosed as AIDS. All of them were drug-naive. The mean CD4 count of all study subjects was 272.1 cells $/ \mu \mathrm{l}$ (95\% CI $60.1-484.1)$. The median CD4 count was $247.5(0-980)$ cells/ $\mu$ l. The mean plasma HIV RNA load was $135,819.4$ copies $/ \mathrm{ml}$. The median plasma HIV RNA load was 44,000 (50-2,300,000).

\section{Prevalence of Transmitted Drug Resistance}

According to the SDRM algorithm, the overall prevalence of HIV drug resistance in the cross-sectional study in Guangxi was 3.2\% (7/216, 95\% CI 0.9-5.5). The prevalence of HIV drug resistance in Baise, Guilin, Hezhou, Qinzhou and Laibin was 4.9 (95\% CI -1.7 to 11.5 ), 2.3 (95\% CI -2.1 to 5.7 ), 4.7 (95\% CI -1.6 to 11.0 ), 2.6 (95\% CI -2.5 to 7.7$)$ and 2.0 (95\% CI -1.9 to 5.9$)$, respectively (fig. 1). Clearly, the highest prevalence is in Baise City and the lowest in Labin City. However, the difference in the prevalence between Baise City and Laibin City is not significant $\left(\chi^{2}=0.031, \mathrm{p}>0.95\right)$.

The prevalence of HIV drug resistance in males and females was $2.6 \%$ (95\% CI 0.06-5.14) and 4.6\% (95\% CI
-0.49 to -9.69$)$, respectively. The difference in the prevalence between males and females is not significant $\left(\chi^{2}=\right.$ $0.109, \mathrm{p}>0.1)$, suggesting that gender is not associated with TDR. The prevalence of HIV drug resistance in HIV-positive persons and AIDS patients is 2.5\% (95\% CI -0.27 to 5.27 ) and $4.3 \%$ (95\% CI $0.20-8.40)$, respectively. The difference in the prevalence between asymptomatic HIV infection and AIDS is not significant $\left(\chi^{2}=0.124\right.$, $\mathrm{p}>0.1$.

\section{Transmitted Drug Resistance-Associated Mutations}

In this study, 7 samples were found to have TDR mutations (table 2), conferring reduced susceptibility to ARV drugs. Three of the TDR mutations (1.4\%, 95\% CI -0.17 to 2.97 ) cause resistance to nucleoside reverse transcriptase inhibitor (NRTI). T69ADNT confers low-level resistance to didanosine (DDI). Both K70R and T215IS confer low-level resistance to stavudine (D4T). K70R also causes intermediate-level resistance to azidothymidine (AZT) but T215IS only causes low-level resistance to AZT. Four of the TDR mutations cause resistance to nonNRTI (NNRTI) (1.9\%, 95\% CI -0.08 to 3.72). Y181C confers high-level resistance to nevirapine (NVP) and intermediate-level resistance to etravirine (ETR) and rilpivirine (RPV). E138G and V179E within individual samples 
Table 2. Virological characteristics of Guangxi treatment naive patients with primary DRM

\begin{tabular}{|c|c|c|c|c|c|c|c|c|c|}
\hline $\begin{array}{l}\text { Sample } \\
\text { ID }\end{array}$ & Gender & $\begin{array}{l}\text { Age, } \\
\text { years }\end{array}$ & $\begin{array}{l}\text { Infection } \\
\text { stage }\end{array}$ & Genotype & $\begin{array}{l}\text { CD4, cells/ } \\
\mathrm{mm}^{3}\end{array}$ & $\begin{array}{l}\text { Viral } \\
\text { loads }\end{array}$ & $\begin{array}{l}\text { Mutation } \\
\text { to PI }\end{array}$ & $\begin{array}{l}\text { Mutation } \\
\text { to NRTI }\end{array}$ & $\begin{array}{l}\text { Mutation } \\
\text { to NNRTI }\end{array}$ \\
\hline BS020 & M & 36 & HIV & CRF08_BC & 424 & 6,200 & & & K103KN \\
\hline GL047 & $\mathrm{F}$ & 63 & AIDS & CRF01_AE & 337 & 11,009 & M46I & & \\
\hline HZ036 & M & 22 & HIV & CRF01_AE & 440 & 42,000 & & & E138G, V179E \\
\hline HZ041 & $\mathrm{M}$ & 32 & AIDS & CRF01_AE & 49 & 280,000 & & T215IS & V106IV \\
\hline
\end{tabular}

Table 3. Prevalence of HIV genotypes by city

\begin{tabular}{llllll}
\hline Cities & n & CRF01_AE & CRF07_BC & CRF08_BC & B \\
\hline Baise & 41 & $12(29.3 \%, 15.4$ to 43.2$)$ & $3(7.3 \%,-0.7$ to 15.3$)$ & $26(63.4 \%, 48.7$ to 78.1$)$ & 0 \\
Guilin & 44 & $38(86.4 \%, 76.3$ to 96.5$)$ & $3(6.8 \%,-0.6$ to 14.2$)$ & $3(6.8 \%,-0.6$ to 14.2$)$ & 0 \\
Hezhou & 43 & $40(93.0 \%, 85.4$ to 100.6$)$ & $2(4.7 \%,-1.6$ to 11.0$)$ & $1(2.3 \%,-2.2$ to 6.8$)$ & 0 \\
Qinzhou & 38 & $24(63.2 \%, 47.9$ to 78.5$)$ & $6(15.8 \%, 4.2$ to 27.4$)$ & $8(21.1 \%, 8.1$ to 34.1$)$ & 0 \\
Laibin & 50 & $43(86.0 \%, 76.4$ to 95.6$)$ & $2(4.0 \%,-1.4$ to 9.4$)$ & $3(6.0 \%,-0.6$ to 12.6$)$ & $2(4.0 \%,-1.4$ to 9.4$)$ \\
\hline Total & 216 & $157(72.7 \%, 66.8$ to 78.6$)$ & $16(7.4 \%, 3.9$ to 10.9$)$ & $41(19.0 \%, 13.8$ to 24.2$)$ & $2(0.9 \%,-0.4$ to 2.2$)$ \\
\hline
\end{tabular}

cause low-level resistance to efavirenz (EFV), ETR, RPV and NVP. K103KN results in high-level resistance to NVP and EFV. Both protease inhibitor (PI) major resistance mutations (M46I) and PI minor resistance mutations (T74S) cause low-level resistance to nelfinavir (NFV) $(1.4 \%, 95 \%$ CI -0.17 to 2.97$)$. Clearly, resistance was found to all drugs of the first-line regimens, except for lamivudine (3TC) and tenofovir (TDF). TDF was added to the free first-line regiment according to the Manual of the National Free Antiretroviral Treatment (3rd version, published in 2012).

\section{Prevalence of HIV Genotypes in Various Cities}

All of the genotypes of HIV in Guangxi are HIV-1. The subtypes are CRF01_AE, CRF07_BC, CRF08_BC and B. The total prevalence of CRF01_AE, CRF07_BC and CRF08_BC is 72.7\% (95 CI 66.8-78.6), 7.4\% (95\% CI 3.9$10.9)$ and $19.0 \%$ (95\% CI 13.8-24.2). Subtype B was only found in Laibin City and accounts for $4.0 \%$ (95\% CI -1.4 to 9.4) of infections in Laibin City. Clearly, subtype CRF01_AE is the dominant subtype in Guangxi. However, the dominant subtypes vary in different sampling sites. Subtype CRF08_BC is the dominant subtype in Baise (63.4\%, 48.7-78.1) while CRF01_AE is the dominant subtype in all other cities. The prevalence of CRF01_
AE in Hezhou is highest (93.0\%, 85.4-100.6) among all the cities in Guangxi (table 3).

TDR could be seen in subtypes CRF08_BC and CRF01_ AE only. The prevalence of TDR in subtype CRF08_BC and CRF01_AE is $7.3 \%(3 / 41)$ and $2.5 \%$ (4/157), respectively. Although the prevalence of TDR of subtype BC-8 is higher, it is not significantly higher $(\mathrm{X}=0.995, \mathrm{p}>0.5)$ than that of other subtypes, suggesting that genotype is not associated with the development of TDR.

\section{Risk Factors for Transmitted HIV Drug Resistance}

In order to search for risk factors for transmitted HIV drug resistance, logistic regression analysis was performed. The factors included in the analysis are gender, ages, CD4 cell counts, viral loads, employment, marriage, education, transmission route and genotypes. No variables were found to be associated with TDR.

\section{Discussion}

The major finding of the study is that the prevalence of TDR HIV in ARV-naive patients in Guangxi remains low according to WHO thresholds, although the scale-up of free ARV was started 8 years ago and, especially, after 
nearly 3 years of the 5-year period anti-HIV campaign of Guangxi Government. Resistance was found to all drugs of the first-line regimens, except for 3TC and TDF. Subtype CRF08_BC is the dominate subtype in Baise while CRF01_AE is the dominate subtype elsewhere in Guangxi. The strengths of the study are that the samples were collected 8 years after beginning the scale-up of free ARV, which is long enough for the emergence of resistance and transmission. The weakness is that there is not enough time for the emergence of resistance and transmission of TDF because this was added into the first-line regiment only a year ago.

It is recommended by WHO that transmitted HIV drug resistance surveys be performed in geographic areas where ART has been available to at least $20 \%$ of eligible individuals for $\geq 3$ years [14]. NFATP was launched in 2002 and scaled up in Guangxi in 2005. According to the national HIV epidemiology and treatment databases, there were 14,484 and 35,264 patients in Guangxi who had received free ART, by the end of June 2010 and January 2013, respectively. Clearly, the number of individuals who had received free ART dramatically increased from since June 2010 under the 5 years anti-HIV programme run by the Guangxi Government, China. In 2008, Su et al. [15] analysed 124 HIV-infected, ARV-naive patients enrolled from Liuzhou and Nanning, Guangxi. The results showed that the prevalence of transmitted HIV drug resistance was $1.6 \%$. In 2009, Li et al. [16] analysed 211 sequences from individuals from different cities within Guangxi and found that the prevalence of TDR was $1.9 \%$. Our study shows that the prevalence of TDR in Guangxi is 3.2\%. Furthermore, Su et al. [15] did not find TDR in NNRTI. Mutations of this type were seen our in study and that of Li et al. [16] with a prevalence of 1.9 and $0.5 \%$, respectively. Clearly, the prevalence of TDR in $\mathrm{HIV}$-infected, ARV-naive patients in Guangxi remains low. However, it is increasing.

It is estimated that there is a 7 - to 10 -year period of latency from HIV infection to AIDS [14]. Su et al. [15] and Li et al. [16] collected samples $4-5$ years after the scale-up of NFATP. It is possible that some of their study subjects contracted HIV before the scale-up. Therefore, their results may not reflect the status after the scale-up. Our samples were collected between December 2012 and January 2013, 8 years after the scale-up. Therefore, our results may reflect the current status.

Extensive use of ARV drugs has led to increasing transmission of HIV variants with DRMs that can be maintained in individuals before initiation of treatment [12]. Patients in countries in which ART had been available for $\geq 5$ years were 1.7 times more likely to have TDR than those living in a country where ART had been available for $<5$ years [17]. The prevalence of transmitted HIVDR is highest in developed countries where ARV drugs are available early, estimated between 8.4 and $22.7 \%$ [18]. The overall rate of TDR in all resource-limited countries was about $6.6 \%$. The reported prevalence of TDR was 5.7\% in Africa, $7.6 \%$ in Asia and $8.4 \%$ in Brazil [17].

The first HIV epidemic in China occurred in 1989 in Dehong prefecture, bordering northern Myanmar. A survey from the prefecture reveals that the prevalence of TDR was $4.3 \%$ after 7 years of the scale-up [19]. Another study from Guangdong province showed that the prevalence of TDR was $6.7 \%$ after 5 years of the scale-up [20]. A report from Henan province, the first province to provide free ARV to HIV-infected individuals in 2003 in China, shows that the prevalence of TDR in the province is $14.3 \%, 8$ years after the scale-up [21]. The difference in the prevalence of TDR between Guangxi and Henan provinces is significant $\left(\chi^{2}=13.178, p<0.001\right)$, although the period from scale-up to sample collection is the same. It may be interesting to search for the factors that led to the difference in the prevalence of TDR between Guangxi and Henan.

According to the Manual of the National Free Antiretroviral Treatment (2nd version, published in 2007), treatments in NFATP were first-line ART regimens consisting of two NRTIs (AZT + DDI or D4T + 3TC) and one NNRTI (NVP or efavirenz (EFV)). AZT, D4T, DDI, and NVP are generically produced in China, whereas 3TC and EFV are branded drugs which became available in $2005[22,23]$. In this study, there are mutations that confer resistance to first-line drugs, except for 3TC. However, the geographic distribution of drug resistance varies. Therefore, the findings in the study are important to guide first-line ART selection.

HIV has extreme genetic variability due to high mutation and recombination rates caused by the reverse transcriptase enzyme [24]. HIV diversity impacts on virtually every aspect of the HIV pandemic, including disease progression and transmission, diagnosis and measurement of viral load, response to HAART, drug resistance, the immune response and vaccine development [25]. Therefore, the information from the surveillance of HIV subtypes and recombination is important. Results from this study show that the dominant subtype in Guangxi is CRF01_AE, the same as Li et al. [16] reported 4 years ago However, the dominant subtype in Baise is subtype CRF08_BC, different from that reported by Li et al. This may be due to the extreme genetic variability of HIV but, 
alternatively, it may also be because of a bias in the study of Li et al. because their sample size was small (11 study subjects only).

TDR surveillance will generate important information to guide first-line ART selection and promote the rational use of ARV drugs by clinicians and policymakers $[11,12]$. Since the Guangxi Government has started the 5-year anti-HIV programme, there will be more and more HIV-infected individuals on free ART. Data from our study showed that the prevalence of TDR in Guangxi is increasing. Therefore, we will continue to survey TDR in the future.

\section{Acknowledgements}

We are indebted to staff members of Centers for Disease Prevention and Control in the cities of Baise, Guilin, Hezhou, Qinzhou, and Laibin, Guangxi, who assisted in recruiting the study subjects and handling the sera. This work was supported by the Ministry of Science and Technology of China (Grant No. 2012ZX10004910) and National Natural Science Foundation of China (Grant No. 30960326/C150501).

\section{Disclosure Statement}

The authors have no conflict of interest to disclsoe.

\section{References}

1 UNAIDS: Report on the Global AIDS Epidemic. Geneva, UNAIDS, 2012. http://relief web.int/report/world/unaids-report-globalaids-epidemic-2012.

2 Hemelaar J: The origin and diversity of the HIV-1 pandemic. Trends Mol Med 2012;18: 182-192.

-3 Karlsson A, Björkman P, Bratt G, Ekvall H, Gisslén M, Sönnerborg A, Mild M, Albert J: Low prevalence of transmitted drug resistance in patients newly diagnosed with HIV-1 infection in Sweden 2003-2010. PLoS One 2012;7:e33484.

-4 Nwobegahay JM, Bessong PO, Masebe TM, Mavhandu LG, Iweriebor BC, Selabe G: Prevalence of antiretroviral drug resistance mutations and HIV-I subtypes among newly-diagnosed drug-naive persons visiting a voluntary testing and counselling centre in northeastern South Africa. J Health Popul Nutr 2011;29: 303-309.

5 Gupta RK, Jordan MR, Sultan BJ, Hill A, Davis DH, Gregson J, Sawyer AW, Hamers RL, Ndembi N, Pillay D, Bertagnolio S: Global trends in antiretroviral resistance in treatment-naive individuals with HIV after rollout of antiretroviral treatment in resource-limited settings: a global collaborative study and meta-regression analysis. Lancet 2012;380: $1250-1258$.

6 Cortez KJ, Maldarelli F: Clinical management of HIV drug resistance. Viruses 2011;3:347378.

7 Readhead AC, Gordon DE, Wang Z, Anderson BJ, Brousseau KS, Kouznetsova MA, Forgione LA, Smith LC, Torian LV: Transmitted antiretroviral drug resistance in New York State, 2006-2008: results from a new surveillance system. PLoS One 2012;7:e40533.

8 The Ministry of Health of China, UNAIDS, WHO: HIV/AIDS Estimate Process in China in 2007. Chin J AIDS STD 2012;18:1-5.

9 Liu W, Liang FX, Wang SS, Chen J: The report of the first confirmed Chinese case with HIV in Guangxi. Guangxi Med J 1993;15:193-194.
10 Chen J, Liang FX, Liang SL, Li RJ, Liu W, Yang JY, Wang SS, Gu YC, He B: An investigation on HIV infection among drug users at Pingxiang City in Guangxi province, Southern China. Chin J AIDS STD 1998;4:97-99.

11 Bennett DE, Myatt M, Bertagnolio S, Sutherland D, Gilks CF: Recommendations for surveillance of transmitted HIV drug resistance in countries scaling up antiretroviral treatment. Antivir Ther 2008;13(suppl 2):25-36.

$\checkmark 12$ Avila-Ríos S, Mejía-Villatoro CR, GarcíaMorales C, Soto-Nava M, Escobar I, Mendizabal R, Girón A, García L, Reyes-Terán G: Prevalence and patterns of HIV transmitted drug resistance in Guatemala. Rev Panam Salud Publica 2011;30:641-648.

13 Bennett DE, Camacho RJ, Otelea D, Kuritzkes DR, Fleury H, Kiuchi M, Heneine W, Kantor R, Jordan MR, Schapiro JM, Vandamme AM, Sandstrom P, Boucher CA, van de Vijver D, Rhee SY, Liu TF, Pillay D, Shafer RW: Drug resistance mutations for surveillance of transmitted HIV-1 drug-resistance: 2009 update. PLoS One 2009; 4:e4724.

14 Schellenberg JJ, Plummer FA: The microbiological context of HIV resistance: vaginal microbiota and mucosal inflammation at the viral point of entry. Int J Inflam 2012;2012: 131243.

15 Su Q, Liang H, Cen P, Bi Z, Zhou P: HIV type 1 subtypes based on the pol gene and drug resistance mutations among antiretroviral-naive patients from Guangxi, Southern China. AIDS Res Hum Retroviruses 2012;28:725728.

16 Li L, Chen L, Liang S, Liu W, Li T, Liu Y, Li H, Bao Z, Wang X, Li J: Subtype CRF01_AE dominate the sexually transmitted human immunodeficiency virus type 1 epidemic in Guangxi, China. J Med Virol 2013;85:388395.

17 Stadeli KM, Richman DD: Rates of emergence of HIV drug resistance in resource-limited settings: a systematic review. Antivir Ther 2013;18:115-123.
18 Hong SY, Nachega JB, Kelley K, Bertagnolio S, Marconi VC, Jordan MR: The global status of HIV drug resistance: clinical and publichealth approaches for detection, treatment and prevention. Infect Disord Drug Targets 2011;11:124-133.

19 Chen M, Ma Y, Duan S, Xing H, Yao S, Su Y, Luo H, Yang L, Chen H, Fu L, Qu A, Ou CY, Jia M, Lu L: Genetic diversity and drug resistance among newly diagnosed and antiretroviral treatment-naive HIV-infected individuals in western Yunnan: a hot area of viral recombination in China. BMC Infect Dis 2012;12:382.

20 Yang C, Liu S, Zhang T, Hou Y, Liu X, Gao Y, Yang G, Wang Z, Chen H, Li M, Zhu Z: Transmitted antiretroviral drug resistance and thumb subdomain polymorphisms among newly HIV type 1 diagnosed patients infected with CRF01_AE and CRF07_BC virus in Guangdong province, China. AIDS Res Hum Retroviruses 2012;28:1723-1728.

21 Li L, Sun B, Zeng H, Sun Z, Sun G, Yang R: Relatively high prevalence of drug resistance among antiretroviral-naive patients from Henan, Central China. AIDS Res Hum Retroviruses 2014;30:160-164.

22 Ruan Y, Xing H, Wang X, Tang H, Wang Z, Liu H, Su B, Wu J, Li H, Liao L, Li J, Wu JW, Shao Y: Virologic outcomes of first-line HAART and associated factors among Chinese patients with HIV in three sentinel antiretroviral treatment sites. Trop Med Int Health 2010;15:1357-1363.

23 Xing H, Wang X, Liao L, Ma Y, Su B, Fu J, He J, Chen L, Pan X, Dong Y, Liu W, Hsi JH, Yang L, Ruan Y, Shao Y: Incidence and associated factors of HIV drug resistance in Chinese HIV-infected patients receiving antiretroviral treatment. PLoS One 2013;8:e62408.

24 Aldrich C, Hemelaar J: Global HIV-1 diversity surveillance. Trends Mol Med 2012;18: 691-694.

25 Hemelaar J: Implications of HIV diversity for the HIV-1 pandemic. J Infect 2013;66:391400. 